\section{COMMENTS BY REVIEWER A}

This manuscript does provide a good example of the manner in which the effects of certain policy changes in a correctional institution might more systematically be evaluated to determine impact. There do, of course, remain a number of problems-mainly these are in respect to lack of relevant and important details and information (e.g., the precise nature of the policy change)-including the remarkable fact that the transfer policy was never explicitly stated or made known to the inmates or to staff. However, I believe that we have to accept these limitations as given in this situation. It is my distinct impression that the authors have done about as much as they possibly could, given the constraints of the situation and facility. I thought the Discussion section was very good and that it quite adequately addressed the weaknesses, threats to validity, and relevance of plausible alternative explanations. Therefore, I would recommend that the revised manuscript be accepted for publication as an example of efforts to analyze retrospectively the effects of policy changes.

\section{REVIEWER B}

It is unlikely that the role of one component of this policy change (interpreted as Timeout from Positive Reinforcement) could ever be convincingly demonstrated with this data. One should, therefore, confine the inference to be drawn from the data to the policy change in toto and not one conceptual piece of it. Furthermore, the importance of the paper is not to recommend this policy change, but rather to exemplify the process of arriving at conclusions from retrospective analysis.

\section{COMMENTS BY REVIEWER C}

The retrospective analysis of data to estimate the effects of an uncontrolled natural event is a process familiar to natural science. Wherever possible, however, the causality between the natural event and the assumed effects is confirmed by systematic experimentation-in which the presumed causal event is explicitly controlled and other possible causes can be thereby eliminated.

The operational phrase is "wherever possible". When causality cannot be established through experimentation in the laboratory or in the field, a conclusion of causality based upon a quasiexperimental analysis of the data surrounding the natural event is tentatively accepted-and experiments thought analogous to the natural phenomenon must be relied upon to strengthen this tentative conclusion. (An example of this is the link between cigarette smoking and lung cancer.)

With the rest of natural science, applied behavior analysis should accept and utilize quasiexperimental analyses for two reasons:

(1) Massively important social "adjustments" are being made every day by leaders and administrators of our society. These adjustments are presently usually as inaccessible to behavioral scientists as are earthquakes and sunspots to physical scientists. Nevertheless, their effects should be evaluated as best they can be-for present benefit to society through the threat of accountability, and future benefit through more substantial social information.

(2) Behavioral scientists will occasionally have the opportunity to influence the timing and target of new social "reforms" and to design and implement increasingly larger social programs. These opportunities for experimental analysis will arise more frequently in the future. The informal quasi-experimental attempts at applying the principles of behavior to human problems attempted by "operant conditioners" in the late 1950s and early 60 s provided the pilot work that allowed the "behavior modifiers" of the 1960s to 
capitalize successfully on the occasional opportunities to assume major responsibility for the education or therapy of deviant persons. Similarily, careful quasiexperimental analyses by "applied behavior analysts" of social programs and large scale innovations now will provide the conceptual and technological pilot work enabling us to capitalize successfully on the opportunities for experimental analyses of social systems in our next stage of evolution. 